\title{
An observational study of abdominal organ involvement detected by ultrasound and computed tomography in children suffering from lymphoreticular malignancy at a tertiary care hospital in Eastern India
}

\begin{abstract}
Introduction: Abdominal organs are usually involved in lymphoreticular malignancies (LRM), and the detection is crucial for Initial staging, determination of the location and extent of disease, and is the hallmark for the choice of treatment. At present, the established radiological technique for staging Hodgkin's disease is computed tomography (CT) and ultrasonography (USG) in our country.

Objective: The objective of the study was to evaluate the pattern of abdominal organ involvement in childhood LRM by USG and CT and to analyze the findings.

Methods: The study included 121 children with newly diagnosed childhood LRM who underwent real time USG and contrast enhanced CT scan. The records of the US and CT scanning were analyzed with respect to size, shape, margins, echogenicity /density pattern of various abdominal organs and lymph nodes to evaluate the extent and pattern of abdominal organ involvement by the disease process.

Results: Out of 121 cases of LRM, US detected significant portal lymphadenopathy in 9 (7.44\%) cases where CT detected enlarged portal nodes in only 3 and missed in 6 cases. However in the retroperitoneal lynphadenopathy CT scored over US, as CT detected $16(13.22 \%)$ cases as against $13(10.74 \%)$ cases detected by US.

Conclusion: In our study we observed abdominal organs are commonly involved at the time of initial presentation in childhood LRM, with diffuse organomegaly being commoner than focal lesions. Abdominal symptoms and GIT involvement were also found more common in NHL patients at their initial presentation. There are no characteristic patterns of involvement either on US or CT which are specific to any individual disease entity. Although organomegaly and focal lesions are not diagnostic, but in a known case of childhood LRM these are highly suggestive of involvement by the existing disease process. There is no significant difference in the detection rate of abdominal organ involvement by US and CT in our study. However US being safer and cheaper, US may be suggested as the primary imaging modality to detect abdominal organ involvement in childhood LRM at initial presentation.
\end{abstract}

Key words: Computed Tomography; lymph reticular malignancy; ultrasonography

\section{Introduction}

The term childhood lymphoreticular malignancy (LRM) embraces two major groups of malignant diseases of the lymphoreticular cell origin in children, including lymphomas-Hodgkin's and non-Hodgkin's types and acute leukemia-lymphoblastic and nonlymphoblastic varieties. These altogether constitute about $35-40 \%$ of all childhood malignancies. ${ }^{[1,2]}$

\begin{tabular}{|l|c|}
\hline \multicolumn{2}{|c|}{ Access this article online } \\
\hline \multirow{2}{*}{$\begin{array}{l}\text { Website: } \\
\text { www.asjo.in }\end{array}$} & Quick Response Code \\
\hline \multirow{2}{*}{$\begin{array}{l}\text { DOI: } \\
\text { 10.4103/2454-6798.180591 }\end{array}$} & \\
&
\end{tabular}

Abhiram Chakrabarti, Manab Nandy ${ }^{1}$, Partha Sarkar ${ }^{1}$, Ananya ChOWdhury ${ }^{1}$, Rafiqul Rahaman ${ }^{2}$ Department of Radiology and Radio Diagnosis, Calcutta National Medical College and Hospital, ${ }^{1}$ Department of Pharmacology, Medical College, Kolkata, ${ }^{2}$ Department of Pediatrics, ICARE Institute of Medical Sciences and Research, Dr. Bidhan Chandra Roy Hospital, Kolkata, West Bengal, India

Address for correspondence: Dr. Manab Nandy, No. 95B, Bidhannagar Road, HUDCO Housing Estate, Block 18, Flat 234, Kolkata - 700 054, West Bengal, India.

E-mail: manabn@gmail.com

This is an open access article distributed under the terms of the Creative Commons Attribution-NonCommercial-ShareAlike 3.0 License, which allows others to remix, tweak, and build upon the work non-commercially, as long as the author is credited and the new creations are licensed under the identical terms.

For reprints contact: reprints@medknow.com

How to cite this article: Chakrabarti A, Nandy M, Sarkar P, Chowdhury A, Rahaman R. An observational study of abdominal organ involvement detected by ultrasound and computed tomography in children suffering from lymphoreticular malignancy at a tertiary care hospital in Eastern India. Asian J Oncol 2016;2:29-34. 
Improved diagnostic techniques and the development of intensive radiotherapy and combination chemotherapy have brought remarkable progress in the management of LRMs. ${ }^{[3]}$ Prognosis and method of treatment in such patients are based on accurate assessment of the extent of the disease. ${ }^{[4-6]}$ According to the Ann-Arbor system of clinical staging of lymphomas, which is extremely important in the management of the patients, the involvement of the abdominal organs is usually the major variable separating stages of the disease. ${ }^{\mid 7]}$

Irrespective of clinical suspicion, all children require abdominal imaging and in most centers abdominal ultrasonography and computed tomography (CT) have replaced all other techniques as part of the staging procedure. ${ }^{[8]}$

Childhood LRMs are commonly encountered in the referral specialized centers like tertiary care institutes..$^{[5,9]}$ To our knowledge, few studies have been done comparing the role of ultrasound (US) and CT in detecting the abdominal organ involvement in childhood LRM. ${ }^{[10,11]}$ US and CT are routinely performed in our institute for the complete evaluation of the disease. We, therefore, conducted this project to study the frequency and pattern of abdominal organ involvement in childhood LRM by US and CT and to analyze the findings on US and CT.

\section{Materials and Methods}

The study was conducted from January 2014 to July 2015, in the Department of Radiodiagnosis, at a Tertiary Care Hospital in Eastern India on 121 children of either sex, below the age of 14 years admitted in the Pediatric Unit of the hospital with new diagnosis of LRM on hematological, bone marrow aspiration or cytological examinations. Real-time US and contrast enhanced CT (CECT) of the abdomen were performed before the start of the specific therapy. Subjects were excluded from the study if they had renal impairment, abnormal thyroid function tests or any history of allergy to intravenous contrast media.

All the patients were subjected to detailed clinical evaluation and laboratory investigations including complete hemogram, renal and thyroid function tests, peripheral blood smear, bone marrow examination, and fine needle aspiration cytology (FNAC) and/or excision biopsy examination of lymph nodes (LNs) or abdominal mass prior to the radiological examinations.

US examination of the abdomen was done on GE RT 3600/4600 and Sonoline SI 450 US scanner using 3.5 and $5.0 \mathrm{MHz}$ sector and/or linear transducers. The scanning was done in both longitudinal and transverse axis with the patient in the supine, prone, and bilateral decubitus positions. Oblique and intercostal scans were obtained as needed. The images were photographed according to their gray scale pattern.

CECT of the abdomen was done in supine position on SOMATOM HiQs (SIEMENS) scanner. Infants and young children were given sedation as and when required. Oral contrast was given orally or through nasogastric tube as $2 \%$ solution of water soluble iodinated contrast agent $(60 \%)$, $45 \mathrm{~min}$ and $15 \mathrm{~min}$ prior to scanning.

The records of the US and CT scanning were analyzed with respect to size, shape, margins, echogenicity/density pattern of various abdominal organs and LNs to evaluate the extent and pattern of abdominal organ involvement by the disease process.

Results were statistically analyzed using Chi-square test and were considered statistically significant if $P \leq 0.05$. Analysis was done by computer software "GraphPad Instat 3" (GraphPad Software).

\section{Observation and Results}

Mild to moderate fever; progressive, discrete, mobile, nontender, firm cervical lymphadenopathy associated with mild to moderate intermittent fever, and abdominal pain and swelling were the commonest presenting symptoms in the cases of LRM.

As seen from Table 1 acute leukemia cases are 51 in numbers. Among them maximum 31 patients are from 6 to 10 years age group. Also maximum 38 patients are the male Childs among acute leukemia group. In Hodgkin's group all 33 patients are male and maximum 18 are from the age group of 6 to 10 years. In Non-Hodgkin's group total numbers of subjects are 37. Among them maximum patients are male 26. Numbers of maximum patients of 20 are from 6 to 10 years age group.

Table 1: Age and sex distribution of the patients

\begin{tabular}{|c|c|c|c|c|c|c|}
\hline \multirow[t]{2}{*}{$\begin{array}{l}\text { Age range } \\
\text { (years) }\end{array}$} & \multicolumn{2}{|c|}{$\begin{array}{c}\text { Acute leukemia }(n=51) \\
n(\%)\end{array}$} & \multicolumn{2}{|c|}{$\begin{array}{l}\text { Hodgkin's disease }(n=33) \\
n(\%)\end{array}$} & \multicolumn{2}{|c|}{$\begin{array}{c}\text { Non-Hodgkin's lymphoma } \\
(n=37) \\
n(\%)\end{array}$} \\
\hline & Male & Female & Male & Female & Male & Female \\
\hline $0-5$ & $8(15.69)$ & $2(3.92)$ & $12(36.36)$ & 0 & $4(10.81)$ & $4(10.81)$ \\
\hline $6-10$ & $22(43.14)$ & $9(17.64)$ & $18(54.54)$ & 0 & $13(35.14)$ & 7 (18.92) \\
\hline $11-14$ & 8 (15.69) & $2(3.92)$ & $3(9.10)$ & 0 & $9(24.32)$ & 0 \\
\hline
\end{tabular}




\section{Abdominal organ involvement in acute leukemia}

Acute leukemia constituted the maximum number $(n=51)(42.15 \%)$ of patients in our study group. Out of these 51 patients, 44 cases were diagnosed to have acute lymphoblastic leukemia (ALL) and 7 cases acute myeloid leukemia (AML).

In the AML group Figure 1, three patients (43\%) revealed hepatosplenomegaly, the remaining four patients $(57 \%)$ did not show any abdominal organ involvement.

In ALL group, hepatomegaly was seen in 37 (84\%) cases followed by splenomegaly in 30 cases $(68 \%)$ are seen in [Figure 2]. There was a discrepancy between the US and CT in detecting the abdominal LNs in various locations. US detected portal LNs in 4 cases $(9.09 \%)$, whereas in 2 (4.55\%) cases, CT was able to delineate enlarged LNs in porta hepatis [Figure 3]. Apparently, CT turned out to be better in detecting retroperitoneal LNs (R.P.L.Ns) as it detected enlarged retroperitoneal lymphadenopathy in 6 (13.64\%) cases as against 4 cases $(9.09 \%)$ by US [Figure 4 ].

The characteristics of enlarged LNs were similar in all the locations. On US, these were multiple, well-defined, discrete, round or ovoid in shape, and of various sizes ranging from 1.5 to $2 \mathrm{~cm}$. These were homogenously hypoechoic in all the cases. On CT, the enlarged LNs were homogenously hypodense in attenuation and showed similar characteristics as on US in terms of location, number, and size. No necrotic appearance or calcifications were seen in the enlarged LNs.

Bilateral renal involvement was seen in $4(9 \%)$ cases. Among them, one patient showed bilateral enlargement of kidneys with homogenous architecture associated with hepatosplenomegaly. Another case in addition to bilateral nephromegaly revealed focal lesions in the kidneys and retroperitoneal lymphadenopathy. The focal parenchymal lesions were homogenously hypoechoic

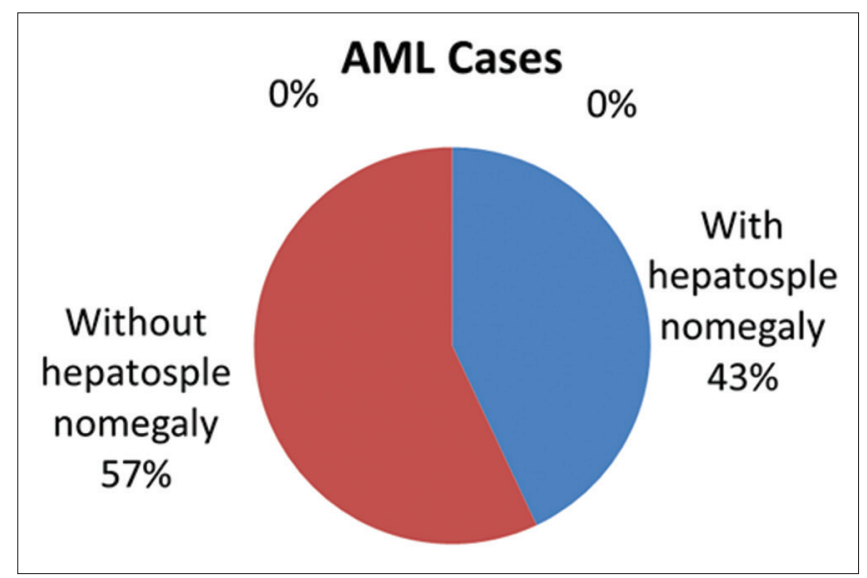

Figure 1: Acute myeloid leukemia with liver and spleen involvement on US and homogenously hypodense on CT. These were multiple, discrete, varying in size $(0.2-3 \mathrm{~cm}$ in diameter) and located diffusely in the renal parenchyma without distorting the pelvicaliceal architecture. However, on CT, the focal lesions were more well-defined than on US. No gastrointestinal tract (GIT) or splenic hilar LN involvement was seen in acute leukemia in our study.

Patterns of involvement of liver and spleen in lymphoma As evident from Table 2, diffuse enlargement of the liver was observed in 21 (63.64\%) and 17 (45.95\%) patients while diffuse splenomegaly was present in 18 (54.55\%) and 7 (18.92\%) patients of Hodgkin's disease (HD) and non-Hodgkin's lymphoma (NHL), respectively.

Six patients, three from each group of HD and NHL demonstrated focal hepatic lesions both on US and CT. On US, all the cases showed homogenously hypoechoic lesions. The lesions were multiple, round or oval, discrete with ill-defined borders ranging in size from 0.2 to $1 \mathrm{~cm}$ and were distributed on both the right and left lobes of the liver with equal frequency. On CT, these focal lesions were similar in number, sizes, and distribution as on US. However, these were well-defined with smooth borders and homogenously hypodense in attenuation. Two cases of focal $L N$ involvement of the liver in HD had hepatomegaly and grossly enlarged R.P.L.Ns and revealed additional focal splenic involvement.

Table 2: Patterns of involvement of liver and spleen in Iymphoma

\begin{tabular}{lccccc}
\hline Type of lymphoma & \multicolumn{2}{c}{ On USG $\boldsymbol{n}(\%)$} & & \multicolumn{2}{c}{ On CT $\boldsymbol{n}(\%)$} \\
\cline { 2 - 3 } \cline { 5 - 6 } & Liver & Spleen & & Liver & Spleen \\
\hline HD $(n=33)$ & & & & \\
$\quad$ Diffuse enlargement & $21(63.64)$ & $18(54.55)$ & $21(63.64)$ & $18(54.55)$ \\
$\quad$ Focal lesion & $3(9.09)$ & $12(36.36)$ & $3(9.09)$ & $12(36.36)$ \\
NHL $(n=37)$ & & & & \\
$\quad$ Diffuse enlargement & $17(45.95)$ & $7(18.92)$ & $17(45.95)$ & $7(18.92)$ \\
$\quad$ Focal lesion & $3(8.11)$ & 0 & $3(8.11)$ & 0 \\
\hline CT - Computed tomography; USG - Ultrasonography; HD - Hodgkin's disease; \\
NHL - Non-Hodgkin's lymphoma
\end{tabular}

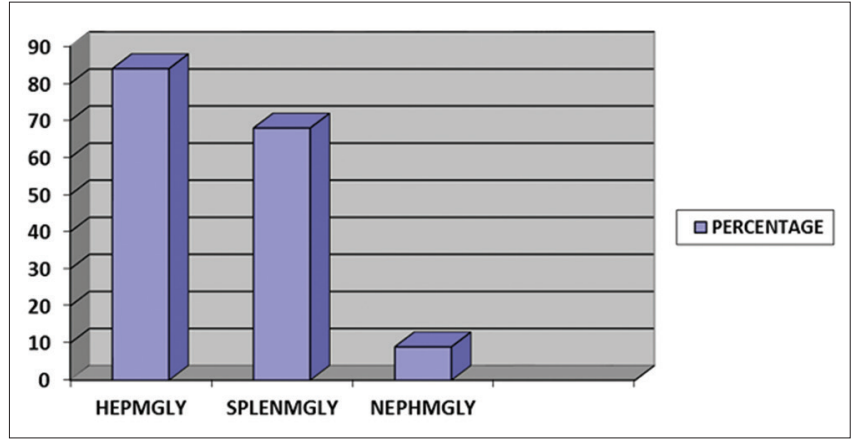

Figure 2: Percentage wise distribution of organ involvement in acute lymphoblastic leukemia 
One case from NHL group was associated with additional GIT involvement.

Focal lesions of spleen were detected on both US and CT in 12 patients $(36.36 \%)$ out of 33 cases of lymphomas at their initial presentation. All the 12 patients were diagnosed to have HD and were associated with splenomegaly. In all the 12 cases, US characteristics of focal lesion were multiple, discrete, oval or round, homogenously hypoechoic, with ill-defined margins and of varying sizes ranging from 0.2 to $1 \mathrm{~cm}$. In one case, there were associated focal lesions in the liver also. On CT, the focal splenic lesions were well-defined, discrete, homogenously hypodense and of attenuation values ranging from 50 to $107 \mathrm{HU}$.

\section{Type of lymph nodes involvement in lymphomas}

In HD, enlarged portal LNs was seen on US in three cases. In one case, only one well-defined ovoid, homogenously hypoechoic LN measuring $1.7 \mathrm{~cm}$ in diameter was noted. Another two cases showed multiple well-defined, discrete, homogenously hypoechoic LNs measuring more than $1 \mathrm{~cm}$ in the portal area. Only one case of portal LN was delineated by CT.

R.P.L.N. enlargement was noted in six cases of HD which were detected on both US and CT. The characteristics of LNs were similar on US and CT. These were multiple, well-defined, discrete as well as confluent, measuring in sizes from 1.5 to $3 \mathrm{~cm}$. The LNs were homogenously hypoechoic on US and homogenously hypodense $(80-95 \mathrm{HU})$ on CT. No areas of calcification or necrotic appearance were noted in the LNs [Figure 5].

Significantly enlarged splenic hilar LNs were present in eight cases of HD which were detected on both US and CT. In five cases, single enlarged (more than $2 \mathrm{~cm}$ ) LN was present in the splenic hilum. This was oval in shape, well-defined homogenously hypoechoic on US, and homogenously hypodense on CT. In the remaining three cases, multiple, well-defined, discrete LNs ranging in size from 1.5 to $2 \mathrm{~cm}$ were noted in the splenic hilum.

In the NHL group, two patients showed multiple, well-defined, ovoid $(0.6-2.4 \mathrm{~cm})$ homogenously hypoechoic LNs on US in the portal area. However, on CT, no portal LN could be detected.

Four cases with NHL showed multiple enlarged R.P.L.N on CT. In three cases, retroperitoneal lymphadenopathy was also detected on US. No LN involvement was detected in the splenic hilum in the patients of NHL. The characteristics of enlarged LN on US and CT were similar in both HD and NHL.

\section{Patterns of renal involvement in lymphomas}

In our study group of sixty cases of lymphomas, five patients showed renal changes at the time of initial presentation. All the five patients had bilateral kidney enlargement and diagnosed to have NHL. No case of HD with renal involvement was found. Four of these five patients revealed bilateral focal renal parenchymal lesions along with enlarged kidneys.

The US findings of the focal parenchymal lesions were similar in all the cases. Lesions were multiple, discrete, ill-defined homogenously hypoechoic with no posterior acoustic enhancement. The focal lesions ranged from 0.2 to $1.5 \mathrm{~cm}$ in diameter, oval or round in shape, and there was no distortion of renal contours. In four patients, the sinus echoes were distorted by the focal lesions. However, there were no hydronephrotic changes in either of the kidneys. All the five cases were associated with enlarged R.P.L.Ns. The CT findings of the renal focal parenchymal lesions were similar in all the cases. These were homogenously hypodense with well-defined margin. The locations of these lesions and the distortion of the collecting systems (observed in one patient) by the nodular masses paralleled the findings of the US examination.

\section{Gastrointestinal tract involvement in lymphoma}

In our study group, out of 60 cases of childhood LRM, gastrointestinal involvement was noted in eight cases, all of which belonged to NHL group. Out of these eight patients, six were male and two female; ranging in age from 3 to 13 years. All of them presented with abdominal pain, mass-abdomen with weight-loss for a period ranging from 15 days to 3 months. One of them had tender bilateral jaw-swelling of 15 days. In all these cases, the diagnosis was made by US-guided FNAC from the bowel pathology. Four out of the eight were histologically diagnosed as Burkitt's type.

Both US and CECT abdomen were able to detect the GIT involvement in all the cases. In 2 cases of Burkitt's lymphoma, the GIT involvement was diffuse in the form of irregular concentric thickening of bowel wall with narrowing of the lumen. Irregular bowel wall thickening extended from the stomach to the rectum. The mesenteric fat also showed diffuse infiltration. It was also associated with multiple confluent retroperitoneal lymphadenopathy and focal renal parenchymal lesions with nephromegaly. Rest 2 cases of Burkitt's lymphoma also revealed, irregularly thickened bowel loops mainly in the right hypochondrium suggestive of proximal small bowel involvement. However, stomach was not involved. They were also associated with retroperitoneal lymphadenopathy. In all the cases, thickened bowel loops appeared as irregular heterogeneous echo texture mass 


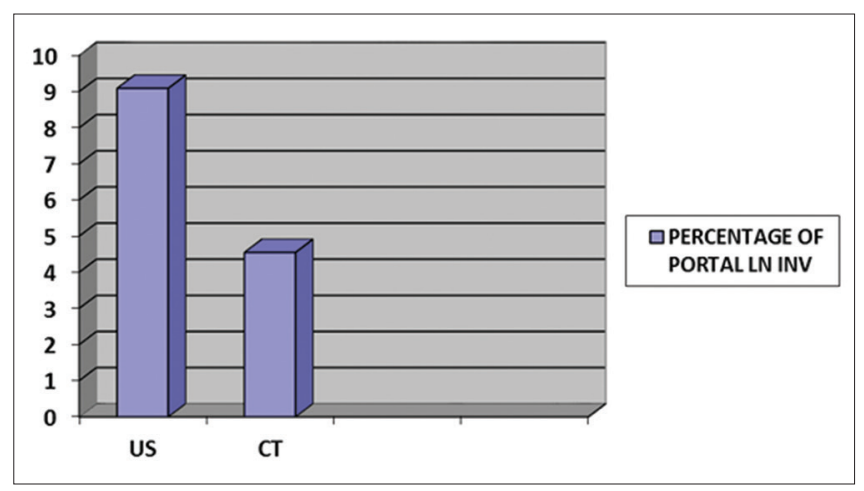

Figure 3: Percentage of portal lymph node involvement in ultrasound and computed tomography in acute lymphoblastic leukemia

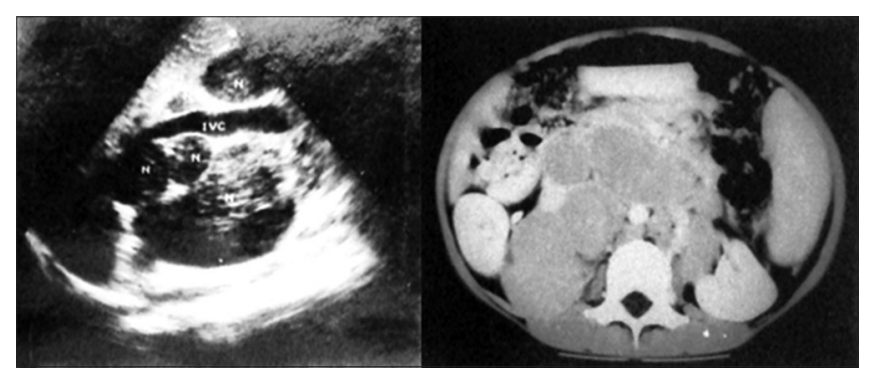

Figure 5: Ultrasonography and axial plane - contrast enhanced computed tomography study of abdomen reveals multiple discrete enlarged retroperitoneal lymph nodes without any internal necrosis or calcification in aortocaval and peri-aortic regions

on US with hyperechoic foci within the mass suggestive of entrapped bowel gas or mucosa. On CT, these were seen as heterogeneous density mass with evidence of contrast agent within them suggestive of bowel pathology.

In the remaining 4 cases, the terminal small bowel and the right colon were involved in the form of large irregular heterogeneous mass lesions causing distortion of terminal ileum, cecum, and ascending colon associated with mesenteric and retroperitoneal lymphadenopathy, as evident in Figure 6a and b.

Abdominal organ involvement in lymphoreticular malignancy: Comparison of detection rate of ultrasound with computed tomography

The detection rate of abdominal organ involvement by US and CT was similar in liver, spleen, kidney, GIT, splenic hilar, and mesenteric LNs involvement are shown in Table 3. However, there was a difference in the detection rate between these two techniques in case of portal and R.P.L.N. involvement. Out of 121 cases of LRM, US detected significant portal lymphadenopathy in 9 (7.44\%) cases where CT detected enlarged portal nodes in only three and missed in 6 cases. However, in the retroperitoneal lymphadenopathy, CT scored over US, as CT detected $16(13.22 \%)$ cases as against $13(10.74 \%)$ cases detected by US.

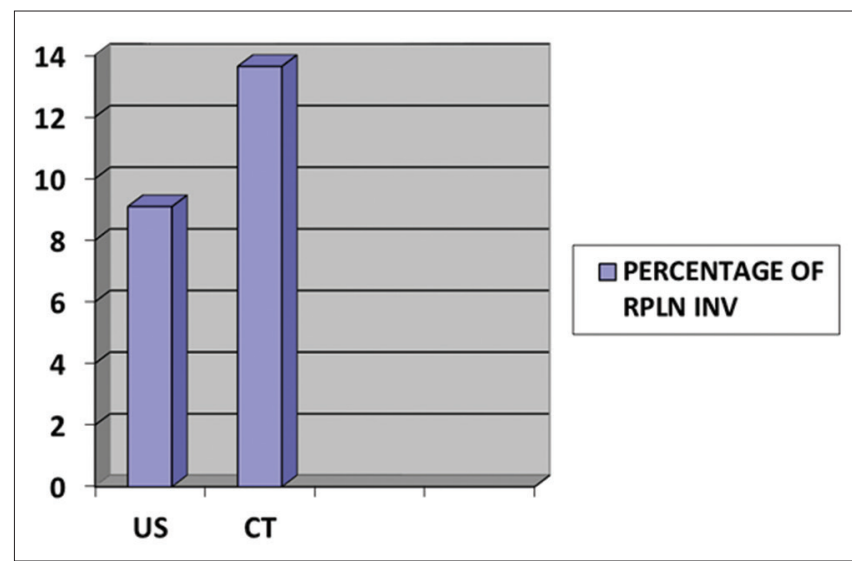

Figure 4: Percentage of retroperitoneal lymph node involvement in ultrasound and computed tomography in acute lymphoblastic leukemia

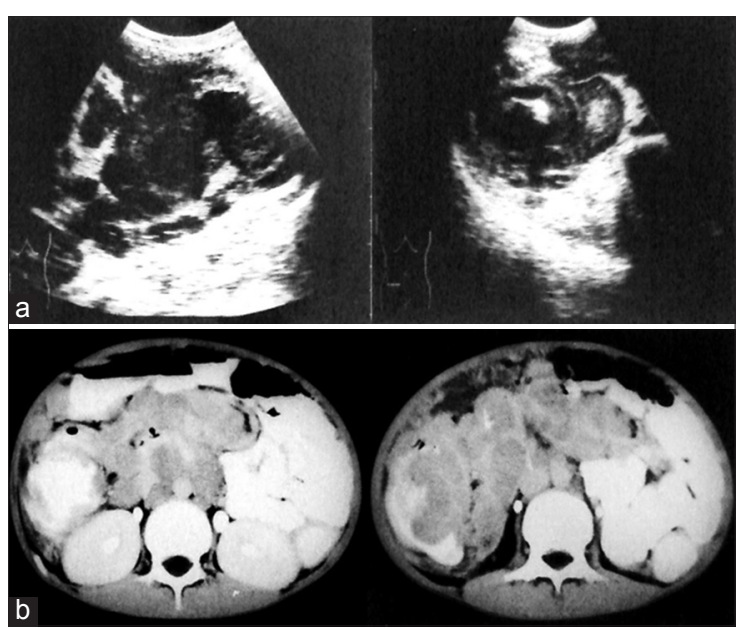

Figure 6: (a) Ultrasound showing enlarged mesenteric lymph nodes and irregular mural thickening of the bowel wall in the right iliac fossa. (b) Contrast enhanced computed tomography of abdomen showing irregular mural thickening of cecum, ascending colon and enlarged mesenteric lymph nodes

Chi-square test for independence: From above table after applying Chi-square test, we find Chi-square: 3.288 , degrees of freedom: $6, P=0.7719$. The row and column variables are not significantly associated.

\section{Discussion}

In our study, we found that abdominal organs are commonly involved at the time of initial presentation in childhood LRM. However, no relationship is present between the duration of presenting symptoms and the extent and type of abdominal organ involvement.

We also observed that abdominal symptoms and GIT involvement are more common in NHL patients at their initial presentation. 
Table 3: Abdominal organ involvement: comparison of detection rate of ultrasonography with computed tomography

\begin{tabular}{lcc}
\hline Organ involvement & On USG $\boldsymbol{n}(\%)$ & On CT $\boldsymbol{n}(\%)$ \\
\hline Liver & $84(69.42)$ & $84(69.42)$ \\
Spleen & $70(57.85)$ & $70(57.85)$ \\
Kidney & $9(7.44)$ & $9(7.44)$ \\
Portal LN & $9(7.44)$ & $3(2.48)$ \\
Retroperitoneal lymph node & $13(10.74)$ & $16(13.22)$ \\
Splenic-hilar and mesenteric LN & $8(6.61)$ & $8(6.61)$ \\
GIT & $8(6.61)$ & $8(6.61)$ \\
\hline
\end{tabular}

CT - Computed tomography; USG - Ultrasonography; GIT - Gastrointestinal tract; LNs - Lymph nodes

Both diffuse organomegaly and focal lesions may be present at the same time; however, organomegaly is commoner than focal lesions. Although organomegaly and focal lesions are not diagnostic, but in a known case of childhood LRM, these are highly suggestive of involvement by the existing disease process. ${ }^{[12]}$ There are no characteristic patterns of involvement either on US or CT, which are specific to any individual disease entity. We also did not find any significant difference in the detection rate of abdominal organ involvement by US and CT in our study. CT is associated with radiation exposure..$^{[13]}$ US may serve as an initial diagnostic test. CT may then be reserved for patients with no diagnostic US results..$^{[14]}$ As US is nonionizing, easily available, and less expensive and has got multi-planar noninvasive imaging capability, US may be recommended as the primary imaging modality to detect abdominal organ involvement in childhood LRM at initial presentation.

\section{Conclusion}

Abdominal organs are commonly involved at the time of initial presentation in childhood LRM. The rate of detection of abdominal organ involvement by US and CT is similar. As US is nonionizing, easily available, and less expensive and has got multi-planar non-invasive imaging capability, it should be recommended as the primary imaging modality followed by $\mathrm{CT}$ if required.

\section{Financial support and sponsorship}

Nil.

\section{Conflicts of interest}

There are no conflicts of interest.

\section{References}

1. Gilham C, Peto J, Simpson J, Roman E, Eden TO, Greaves MF, et al. Day care in infancy and risk of childhood acute lymphoblastic leukaemia: Findings from UK case-control study. BMJ 2005;330:1294.

2. Jemal A, Siegel R, Xu J, Ward E. Cancer statistics, 2010. CA Cancer J Clin 2010;60:277-300.

3. Radich JP. How I monitor residual disease in chronic myeloid leukemia. Blood 2009;114:3376-81.

4. Kaufman M, Rubin J, Rai K. Diagnosing and treating chronic lymphocytic leukemia in 2009. Oncology (Williston Park) 2009;23:1030-7.

5. Ribeiro RC, Pui CH. Saving the children - Improving childhood cancer treatment in developing countries. N Engl J Med 2005;352:2158-60.

6. Lorenzi MF, Xie L, Rogers PC, Pritchard S, Goddard K, McBride ML. Hospital-related morbidity among childhood cancer survivors in British Columbia, Canada: Report of the childhood, adolescent, young adult cancer survivors (CAYACS) program. Int J Cancer 2011;128:1624-31.

7. Lister TA, Crowther D, Sutcliffe SB, Glatstein E, Canellos GP, Young RC, et al. Report of a committee convened to discuss the evaluation and staging of patients with Hodgkin's disease: Cotswolds meeting. J Clin Oncol 1989;7:1630-6.

8. Vázquez E, Lucaya J, Castellote A, Piqueras J, Sainz P, Olivé T, et al. Neuroimaging in pediatric leukemia and lymphoma: Differential diagnosis. Radiographics 2002;22:1411-28.

9. Greenlee RT, Murray T, Bolden S, Wingo PA. Cancer statistics, 2000. CA Cancer J Clin 2000;50:7-33.

10. Rajaraman P, Simpson J, Neta G, Berrington de Gonzalez A, Ansell P, Linet MS, et al. Early life exposure to diagnostic radiation and ultrasound scans and risk of childhood cancer: Case-control study. BMJ 2011;342:d472.

11. The United Kingdom Childhood Cancer Study: Objectives, materials and methods. UK Childhood Cancer Study Investigators. Br J Cancer 2000;82:1073-102.

12. Pritchard-Jones K, Sullivan R. Children with cancer: Driving the global agenda. Lancet Oncol. 2013;14:189-91.

13. Brenner DJ, Hall EJ. Computed tomography - An increasing source of radiation exposure. N Engl J Med 2007;357:2277-84.

14. Laméris W, van Randen A, van Es HW, van Heesewijk JP, van Ramshorst B, Bouma WH, et al. Imaging strategies for detection of urgent conditions in patients with acute abdominal pain: Diagnostic accuracy study. BMJ 2009;338:b2431. 